\title{
Interdisciplinariedad e internacionalización. Una obertura para catorce textos sobre Educación
}

Se presenta el número 3 de Creativity and Educational Innovation Review [CEIR] cuyos temas principales son la interdisciplinariedad y la internacionalización. El número 3 era considerado en la época medieval símbolo de perfección porque representaba a la Trinidad, el dogma central sobre la naturaleza de la divinidad en el mundo cristiano occidental. En la actualidad, 3 son las misiones de las universidades en el Espacio Europeo de Educación Superior, la docencia, la investigación y 'la tercera misión', considerada la transferencia del conocimiento generado para incrementar el desarrollo socio-económico y cultural de su entorno.

No se quiere decir con esto, ni que esta revista que ahora se presenta, sea perfecta -aunque por qué no desearlo- ni que vaya a favorecer su entorno socio-económico y cultural -algo que también sería deseable-. Descendiendo pues de las esferas de las teorías a la realidad circundante, se ofrece a los lectores un grano de arena en la inmensa playa del conocimiento. Este tercer número de CEIR consolida el espacio que ocupa esta revista dentro del Instituto de Creatividad e Innovaciones Educativas de la Universitat de València.

El principal objetivo del presente número de 2019 es ofrecer tanto a la comunidad académica como a los profesionales de la educación y agentes educativos, culturales y sociales una vía de transferencia de los conocimientos generados mediante la investigación en el ámbito universitario y un mecanismo de difusión de los mismos.

El contenido de los textos es interdisciplinar, resultado de las líneas en las que se investiga en el Instituto, la Creatividad, la Educación y la Innovación desde las áreas de trabajo de todos los profesionales que lo integran, la Sociología, la Educación Artística, la Estética o la Psicología, todas ellas dentro del ámbito de las Ciencias Sociales y Humanidades. Además, la cobertura es internacional debido a la red de relaciones que el Instituto y sus miembros tienen con investigadores fuera de las fronteras españolas.

En la primera sección,Dialogando con, se invita a la lectura de una entrevista que lleva por título La sociabilidad humana y la tríada neurociencia cognitiva, psicología experimental y biología evolucionaria, realizada a Ángles Lizón por Judith Muñoz Saavedra, profesora de la Universitat Autònoma de Barcelona. La doctora en Sociología de prestigio internacional Ángeles Lizón ha trabajado en varias universidades americanas [en Canadá y México] así como en la Universitat Autònoma de Barcelona y ha publicado en fechas recientes un libro sobre la caleidoscópica capacidad social de los humanos y la evolución de sus emociones sociales. En este texto, se analizan sus interesantes ideas sobre los modelos teóricos innovadores que abren nuevas perspectivas para ciencia social y cognitiva.

En la sección texto invitado se muestra un estudio realizado sobre la Formación Profesional Dual en el panorama español con un análisis sobre el caso valenciano titulado Understanding cultural barriers and opportunities for developing new apprenticeship programmes. Resultan de especial interés las entrevistas realizadas a diversos actores de la formación profesional en Valencia, así como los resultados obtenidos en este informe. El estudio ha sido llevado a cabo por un equipo multidisciplinar entre los que se encuentran Graham Attwel, director de Pontydysgu, 
una organización de investigación independiente, y profesor en el Institute for Employment Research de la Universidad de Warwick en Reino Unido; la investigadora Ana García Muñoz y el profesor de Psicología Social José Luis García Molinade la Universidad Complutense de Madrid.

En la sección de artículos se han reunido ocho textos, que se han agrupado de forma ternaria alternada con un grupo binario, obteniendo de esta forma una estructura simétrica. Comienza esta sección con tres textos de autoras brasileñas, dos en portugués y uno castellano, que ofrecen diferentes perspectivas y nuevos paradigmas desde la mixtura entre las áreas de la Sociología, los estudios de género y la empleabilidad tras la Educación Superior. En primer lugar, Quando o pesquisador se torna personagem da trama: fragmentos de experièncias, una reflexión dentro de las historias de vida a cargo de la profesora Emilia Maria da Trindade Prestes de la Universidade Federal da Paraíba [Brasil). En segundo lugar, un texto de Luciana Prestes, profesora de Lengua y Literatura española y portuguesa en la Middle Tennessee State University en Estados Unidos de América, titulado Construyendo nuevos paradigmas socioeducativos e identitarios: el cabello como representación de lucha y reconocimiento de la mujer afrodescendiente, en el que analiza el recorrido y la lucha protagonizada por las mujeres afrodescendientes por ejercer el derecho a asumir su identidad reflejada en sus rasgos característicos. El siguiente artículo Entre a tradição e inovação da prática profissional do psicólogo: considerações sobre atuação dos egressos da Universidade Federal de Santa Catarina - Brasil, las autoras Valéria De Bettio Mattos y Gabriela Amâncio de Souza de la Universidad Federal de Santa Catarina en Brasil muestran la investigación realizada con los estudiantes de Psicología de su universidad y su incorporación al mercado de trabajo.

Le siguen dos artículos fruto de estudios relacionados con innovaciones educativas mediante el uso de las tecnologías, en primer lugar, La robótica en la enseñanza de las ciencias en Educación Primaria, una experiencia con 'Bee-Bot', a cargo de la profesora de Didáctica de las Ciencias Experimentales de la Universitat de València Amparo Hurtado Soler y su alumna de máster Natalia Santamaría Peris. En este estudio se utiliza la robótica, un recurso interdisciplinar que promueve el desarrollo de la creatividad y las competencias digitales, para incrementar el aprendizaje de las ciencias naturales en Educación Primaria. El siguiente texto titulado Favorecer el proceso de enseñanza-aprendizaje en educación musical mediante el uso de videojuegos educativos, corre a cargo de Sonsoles Ramos Ahijado y Ana María Botella Nicolás, profesoras de Didáctica de la Expresión Musical de las universidades de Salamanca y Valencia, respectivamente. Ellas han realizado un estudio con futuros docentes en formación en colaboración con alumnado de Educación Primaria para favorecer el aprendizaje del folclore con nuevas tecnologías.

Cierra la revista otro grupo de tres artículos dentro ámbito de la Didáctica de las Artes Plásticas y Visuales, escritos dos en lengua valenciana y uno en castellano. El primero, L'assaig audiovisual com a procés d'exploració crítica. Indagació en les seues potències educatives, fruto del trabajo fin de máster de Vicent Barrachina Sánchez en el que explora las posibilidades pedagógicas de diversos aspectos interdisciplinares presentes en el cine de nuestra cultura actual. Le sigue otro estudio en la misma línea, en el que se analizan materiales audiovisuales como fuente para la identificación del colectivo adolescente, titulado Percepciones y representaciones de la figura heroica e incidencias identitarias en el colectivo adolescente: un estudio de caso desde la Educación Artística, a cargo de Raúl Sol Jódar. Concluye esta sección el estudio realizado sobre L'impacte de la pornografia en l'Educació Primària i la carencia de formació del professorat en materia d'educació sexual, realizado por Pere Gisbert Taberner. Este trabajo saca a la luz la relación divergente entre la educación sexual en las escuelas centrada en la biología y la social focalizada en la pornografía 
y hace hincapié en la necesidad de una reflexión al respecto, así como una mejora en la educación sexual desde edades tempranas para lo cual es necesaria una formación del profesorado.

En la sección de reseñas, se ofrecen información sobre tres encuentros internacionales que han tenido lugar recientemente, dos en la Universitat de València y uno en Minsk [Bielorrusia] y un comentario crítico de un artículo sobre La igualdad de género en la escuela según Mar Venegas, realizado por Laura Peris Reig, doctoranda en la Universidad Complutense de Madrid.

Los congresos internacionales que se han desarrollado en Valencia han sido sobre El patrimonio musical de la Corona de Aragón, 1418-1707: estado de la investigación, transferencia de conocimiento y retos en el s. XXI, comentado por Mireya Royo Conesa, doctora en Musicología por la Universidad de Oviedo y Cultural Festivals Organization and Management: New Challenges in the Digital Age?, glosado por María Devesa Fernández, profesora de Economía Aplicada en la Universidad de Valladolid.

El tercer evento ha sido la conferencia final de un proyecto Erasmus + estructural que ha impulsado las competencias y el sistema del Espacio Europeo de Educación Superior en Bielorrusia y que ha estado coordinado por Adela García-Aracil desde el Instituto de Gestión de la Innovación y del Conocimiento INGENIO, un centro mixto entre el Consejo Superior de Investigaciones Científicas y la Universitat Politècnica de València. La reseña del evento final del proyecto Erasmus + Fostering Competencies Development in Belarusian Higher Education,ha sido realizada por Danguole Bylaitè-Šalavèjienè de la Vytautas Magnus University en Vilnius [Lituania).

Les dejamos con los textos, no sin antes agradecer a la veintena de autores que han enviado sus propuestas, así como a los revisores y revisoras que han colaborado son su saber y tiempo enevaluar los textos y ofrecer propuestas de mejora. Un agradecimiento especial merece el profesor José Beltrán Llavador de la Universitat de València y Francesc J. Hernàndez Dobón, director del Instituto de la Creatividad e Innovaciones Educativas por su impulso en este número. El resultado de la colaboración de todos y todas se lo ofrecemos a continuación, esperamos que sea de su agrado.

Rosa Isusi-Fagoaga 